\title{
The immunology of a successful pregnancy
}

\section{Euroscicon 2013, Pregnancy Summit: The Immunology of a Successful Pregnancy 16 October 2013, London, UK}

The Immunology of a Successful Pregnancy conference brought together scientists and clinicians with an interest in reproductive immunology, with the aim of evaluating some of the 'classic concepts' of pregnancy immunology. A range of talks took place on the day, with the aim of improving our understanding of immunity during pregnancy to benefit both mothers and fetuses in different clinical scenarios.

After a brief welcome note by the host and chair, Rupsha Fraser from the Reproductive and Cardiovascular Disease Research Group, Division of Biomedical Sciences at St George's, University of London, UK, then gave the first presentation. In her talk, she indicated that a successful pregnancy is dependent on efficient placentation and remodeling of maternal uterine vessels (spiralarteries) to allow sufficient oxygen and nutrients to be delivered to the developing fetus. Decidual natural killer (dNK) cells and macrophages (dMФs) accumulate around spiral arteries in early pregnancy and are present during uterine spiral artery remodeling. Fraser further explained that in their study, they had modeled the cellular interactions at the maternal-fetal interface, providing the first demonstration of a functional role of dNK cells in influencing vascular cells, and a potential mechanism contributing to impaired vessel remodeling in pregnancies with a higher uterine artery resistance was presented. Phenotypes of $\mathrm{dM} \Phi$ s that may be present during these events, their roles in the first trimester of pregnancy and spiral artery remodeling, as well as the effects of dNK-derived factors on $\mathrm{d} M \Phi$ polarization were also presented. The second speaker of the day was Lynne Sykes, NIHR Academic Clinical Fellow, Imperial
College London, UK. She highlighted the fact that successful pregnancy is dependent on a carefully balanced modification of the maternal immune response so as to tolerate the semi-allogeneic fetus whilst maintaining protection from harmful pathogens. During term labor, a physiological activation of the immune system occurs, which leads to uterine contractility, fetal membrane rupture and cervical remodeling. However, premature activation of the immune response, often in association with infection, is the most common identifiable cause of preterm labor. Inflammation is also associated with adverse neonatal outcomes, independent of prematurity. The role of inflammation in term and preterm labor, and the potential for anti-inflammatories in the prevention of inflammation-/infection-induced preterm labor was highlighted. That was followed by a midmorning break, poster exhibition and trade show. After the break, oral presentations continued. The session commenced with Sean Wattegedera, a Research Scientist at Moredun Research Institute, Penicuik, UK, presenting. His presentation gave suggestive evidence that the health and welfare of ruminants is key to providing safe and sustainable food for human consumption. Numerous pathogens cause reproductive losses in most sheep-rearing

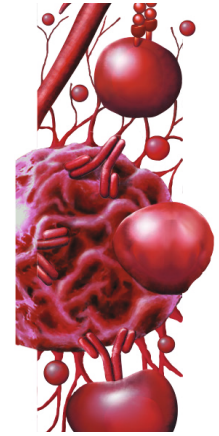

Louisa Tsweleng

Advanced Nursing Science Department, School of Health, University of Venda, University Road, Thohoyandou, Limpopo Province, 0950, South Africa Tel.: +27 159628000 ext. 8679

Fax: +2715962 4749

louisa.tsweleng@univen.ac.za 
countries worldwide and Chlamydia abortus is the most common cause of diagnosed ovine abortion in the UK. Animals can be infected prior to pregnancy and pathogenesis of disease appears to be intimately linked with the progression of pregnancy. To improve on our control measures, it is important to better understand the maternal and fetal immune responses during pregnancy. Data from aspects covering these areas were also presented. That was followed by a talk by Lisa EELO Lashley who discussed the research she conducted together with Marie-Louise P van der Hoorn, Geert Haasnoot, Dave Roelen and Frans HJ Claas, Leiden, The Netherlands. The talk highlighted that a significantly higher level of epitope mismatches was observed for pregnancies after oocyte donation. Furthermore, they found a significantly higher incidence of HLA antibody production in women conceiving after oocyte donation $(56 \%, \mathrm{p}<0.0001)$. A multivariate, backward-stepwise model analysis of the remaining variables showed that epitope and HLA mismatching had a significant influence on antibody formation. Antibody formation was positively correlated with the number of fetomaternal antigen (Spearman's rho 0.84, $\mathrm{p}<0.0001$ ) and epitope mismatches (Spearman's rho 0.87, $\mathrm{p}<0.0001$ ).

The next presentation thereafter was made by Selma Boulenouar on a study she conducted in collaboration with Amanda Sterruzzi-Perri, Hong-Wa Yung, Louise Gaynor, Steve Charnock-Jones, Abby Fowden, Graham Burton and Francesco Colucci from the Department of Obstetrics and Gynaecology, University of Cambridge, UK. The talk gave suggestive evidence that natural killer cell-deficient females (Rag2 $2^{-1} 112 \mathrm{rg}^{-{ }_{-}}$and E4BP4 ${ }^{-/}$) exhibit impaired placental efficiency, as indicated by the significantly reduced fetal/placental ratio generated by smaller fetuses and larger placentae. Structural and morphological analysis of these placentae at term showed enlarged labyrinthine and functional zones, increases maternal blood space and the formation of precipitates in trophoblastic cells. E4BP4 ${ }^{-1-}$ mice exhibited defective spiral artery remodeling, which corroborates previous published data from $\mathrm{Rag} 2^{-1-}$ $112 \mathrm{rg}^{-/-}$mice. Other structural abnormalities observed at midgestation in $\mathrm{E} 4 \mathrm{BP}^{-/-}$mice included increased trophoblast thickness, and decreased MLAp size. This was followed by a lunch break, poster exhibition and trade show, which was followed by a discussion session. The discussion session was an informal question and answer session. Audience/delegates got an opportunity to get advice and opinion from experts in various fields. The session involved not only questions about specific talks, which could be asked after the speaker session, but discussions from general topics or specific issues. After the discussion, a presentation was given by Dilly OC Anumba, a Professor of Obstetrics and Gynecology and an Honorary Consultant Obstetrics and Fetomaternal Medicine Subspecialist at the University of Sheffield, UK. His presentation summarized the immunological basis of vaccinations during pregnancy, highlighting routine and indicated vaccines, and the rationale for their administration. The evidence base for the recommended vaccines during pregnancy in the UK was outlined and areas of uncertainty discussed including an outline of contraindicated vaccines. This was followed by afternoon tea, a poster session and trade show. Thereafter Tess Meuleman, Leiden University of Medical Center, The Netherlands, presented her research. The research was carried out in collaboration with $\mathrm{E}$ van Beelen, LAJ van der Westerlaken, M Eikmans and FHJ Claas. It evidenced that seminal fluid may play a role in priming the maternal system before implantation and therefore helping to create a tolerogenic environment at the implantation site leading to normal pregnancy. To investigate the influence of seminal fluid on maternal peripheral blood mononuclear cells, mixed lymphocyte cultures were performed. Seminal fluid is capable of inducing proliferation of $\mathrm{T}$ cells and it seems that these cells have a more regulatory phenotype. Failure of this immunoregulatory function or less exposure to semen may be the underlying cause in abnormal implantation and lead to complicated pregnancies. This was followed by the last presentation of the day, conducted by Philip Lowry, Emeritus Professor at the University of Reading, UK. The talk gave suggestive evidence that the poorly implanted placenta secretes neurokinin B to correct the associated ischemia, but high concentrations then stimulate all three neorokinin receptors in the mother's circulation, probably causing many of the symptoms of pre-eclampsia. Subsequently it has been found that the placenta post-translationally modifies its own neurokinin $\mathrm{B}$ along with procorticotropin-releasing factor, proactivin, profollistatin and prohemokinin with a moiety containing phosphocholine, a group originally found on certain secreted parasitic proteins that endowed them with immune-inhibiting properties. Thus, the placenta may be utilizing the same survival mechanism as some parasites, attenuating immune surveillance by the mother, and thus avoiding rejection.

\section{Conclusion}

This 1-day conference held in London (UK) was attended by researchers from the UK and all around the world. It was inspiring, stimulating, exciting and 
highly educative and informative. Fascinating presentations were made by students as well as senior researchers on the immunology of a successful pregnancy and the mechanisms and technologies that can be used to enhance it. Problems and pathologies that can occur, when the normal pattern is not realized or achieved, were also highlighted.

Euroscicon's The 2014 Pregnancy Summit will take place from 6-8 October 2014 [1].

\section{Financial \& competing interests disclosure}

The author has no relevant affiliations or financial involvement with any organization or entity with a financial interest in or financial conflict with the subject matter or materials discussed in the manuscript. This includes employment, consultancies, honoraria, stock ownership or options, expert testimony, grants or patents received or pending, or royalties.

No writing assistance was utilized in the production of this manuscript.

\section{Reference}

1 Euroscicon. The 2014 Pregnancy Summit. www.regonline.co.uk/Pregnancy2014 